\title{
Introduction to Matroids ${ }^{1}$
}

\author{
Grzegorz Bancerek \\ Białystok Technical University \\ Poland
}

\author{
Yasunari Shidama \\ Shinshu University \\ Nagano, Japan
}

Summary. The paper includes elements of the theory of matroids [23]. The formalization is done according to [12].

MML identifier: MATROIDO, version: $\underline{7.9 .03 \quad 4.108 .1028}$

The articles [7], [22], [17], [15], [8], [5], [6], [19], [9], [3], [2], [4], [1], [21], [11], [20], [18], [16], [10], [13], and [14] provide the terminology and notation for this paper.

\section{Definition by Independent Sets}

A subset family structure is a topological structure.

Let $M$ be a subset family structure and let $A$ be a subset of $M$. We introduce $A$ is independent as a synonym of $A$ is open. We introduce $A$ is dependent as an antonym of $A$ is open.

Let $M$ be a subset family structure. The family of $M$ yielding a family of subsets of $M$ is defined as follows:

(Def. 1) The family of $M=$ the topology of $M$.

Let $M$ be a subset family structure and let $A$ be a subset of $M$. Let us observe that $A$ is independent if and only if:

(Def. 2) $A \in$ the family of $M$.

Let $M$ be a subset family structure. We say that $M$ is subset-closed if and only if:

(Def. 3) The family of $M$ is subset-closed.

\footnotetext{
${ }^{1}$ This article was done under the Agreement of Cooperation between Białystok Technical University and Shinshu University.
} 
We say that $M$ has exchange property if and only if the condition (Def. 4) is satisfied.

(Def. 4) Let $A, B$ be finite subsets of $M$. Suppose $A \in$ the family of $M$ and $B \in$ the family of $M$ and $\operatorname{card} B=\operatorname{card} A+1$. Then there exists an element $e$ of $M$ such that $e \in B \backslash A$ and $A \cup\{e\} \in$ the family of $M$.

One can check that there exists a subset family structure which is strict, non empty, non void, finite, and subset-closed and has exchange property.

Let $M$ be a non void subset family structure. One can verify that there exists a subset of $M$ which is independent.

Let $M$ be a subset-closed subset family structure. One can verify that the family of $M$ is subset-closed.

We now state the proposition

(1) Let $M$ be a non void subset-closed subset family structure, $A$ be an independent subset of $M$, and $B$ be a set. If $B \subseteq A$, then $B$ is an independent subset of $M$.

Let $M$ be a non void subset-closed subset family structure. Note that there exists a subset of $M$ which is finite and independent.

A matroid is a non empty non void subset-closed subset family structure with exchange property.

One can prove the following proposition

(2) For every subset-closed subset family structure $M$ holds $M$ is non void iff $\emptyset \in$ the family of $M$.

Let $M$ be a non void subset-closed subset family structure. Note that

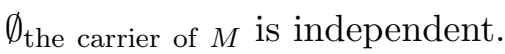

The following proposition is true

(3) Let $M$ be a non void subset family structure. Then $M$ is subset-closed if and only if for all subsets $A, B$ of $M$ such that $A$ is independent and $B \subseteq A$ holds $B$ is independent.

Let $M$ be a non void subset-closed subset family structure, let $A$ be an independent subset of $M$, and let $B$ be a set. One can check the following observations:

* $A \cap B$ is independent,

* $B \cap A$ is independent, and

* $A \backslash B$ is independent.

Next we state the proposition

(4) Let $M$ be a non void non empty subset family structure. Then $M$ has exchange property if and only if for all finite subsets $A, B$ of $M$ such that $A$ is independent and $B$ is independent and card $B=\operatorname{card} A+1$ there exists an element $e$ of $M$ such that $e \in B \backslash A$ and $A \cup\{e\}$ is independent. 
Let $A$ be a set. We introduce $A$ is finite-membered as a synonym of $A$ has finite elements.

Let $A$ be a set. Let us observe that $A$ is finite-membered if and only if:

(Def. 5) For every set $B$ such that $B \in A$ holds $B$ is finite.

Let $M$ be a subset family structure. We say that $M$ is finite-membered if and only if:

(Def. 6) The family of $M$ is finite-membered.

Let $M$ be a subset family structure. We say that $M$ is finite-degree if and only if the conditions (Def. 7) are satisfied.

(Def. 7)(i) $\quad M$ is finite-membered, and

(ii) there exists a natural number $n$ such that for every finite subset $A$ of $M$ such that $A$ is independent holds card $A \leq n$.

Let us note that every subset family structure which is finite-degree is also finite-membered and every subset family structure which is finite is also finitedegree.

\section{EXAMPLES}

Let us note that there exists a set which is mutually-disjoint and non empty and has non empty elements.

The following propositions are true:

(5) For all finite sets $A, B$ such that $\operatorname{card} A<\operatorname{card} B$ there exists a set $x$ such that $x \in B \backslash A$.

(6) For every mutually-disjoint non empty set $P$ with non empty elements holds every choice function of $P$ is one-to-one.

Let us mention that every discrete subset family structure is non void and subset-closed and has exchange property.

Next we state the proposition

(7) Every non empty discrete topological structure is a matroid.

Let $P$ be a set. The functor ProdMatroid $P$ yields a strict subset family structure and is defined by the conditions (Def. 8).

(Def. 8)(i) The carrier of ProdMatroid $P=\bigcup P$, and

(ii) the family of ProdMatroid $P=\left\{A \subseteq \cup P: \wedge_{D \text { :set }}(D \in P \Rightarrow\right.$ $\left.\left.\bigvee_{d: \text { set }} A \cap D \subseteq\{d\}\right)\right\}$.

Let $P$ be a non empty set with non empty elements. One can verify that ProdMatroid $P$ is non empty.

Next we state the proposition

(8) Let $P$ be a set and $A$ be a subset of ProdMatroid $P$. Then $A$ is independent if and only if for every element $D$ of $P$ there exists an element $d$ of $D$ such that $A \cap D \subseteq\{d\}$. 
Let $P$ be a set. One can verify that ProdMatroid $P$ is non void and subsetclosed.

Next we state two propositions:

(9) Let $P$ be a mutually-disjoint set and $x$ be a subset of ProdMatroid $P$. Then there exists a function $f$ from $x$ into $P$ such that for every set $a$ such that $a \in x$ holds $a \in f(a)$.

(10) Let $P$ be a mutually-disjoint set, $x$ be a subset of ProdMatroid $P$, and $f$ be a function from $x$ into $P$. Suppose that for every set $a$ such that $a \in x$ holds $a \in f(a)$. Then $x$ is independent if and only if $f$ is one-to-one.

Let $P$ be a mutually-disjoint set. Observe that ProdMatroid $P$ has exchange property.

Let $X$ be a finite set and let $P$ be a subset of $2^{X}$. One can check that ProdMatroid $P$ is finite.

Let $X$ be a set. Observe that every partition of $X$ is mutually-disjoint.

One can check that there exists a matroid which is finite and strict.

Let $M$ be a finite-membered non void subset family structure. Observe that every independent subset of $M$ is finite.

Let $F$ be a field and let $V$ be a vector space over $F$. The matroid of linearly independent subsets of $V$ is a strict subset family structure and is defined by the conditions (Def. 9).

(Def. 9)(i) The carrier of the matroid of linearly independent subsets of $V=$ the carrier of $V$, and

(ii) the family of the matroid of linearly independent subsets of $V=\{A \subseteq$ $V: A$ is linearly independent $\}$.

Let $F$ be a field and let $V$ be a vector space over $F$. Note that the matroid of linearly independent subsets of $V$ is non empty, non void, and subset-closed.

Let $F$ be a field and let $V$ be a vector space over $F$. Observe that there exists a subset of $V$ which is linearly independent and empty.

The following three propositions are true:

(11) Let $F$ be a field, $V$ be a vector space over $F$, and $A$ be a subset of the matroid of linearly independent subsets of $V$. Then $A$ is independent if and only if $A$ is a linearly independent subset of $V$.

(12) Let $F$ be a field, $V$ be a vector space over $F$, and $A, B$ be finite subsets of $V$. Suppose $B \subseteq A$. Let $v$ be a vector of $V$. Suppose $v \in \operatorname{Lin}(A)$ and $v \notin \operatorname{Lin}(B)$. Then there exists a vector $w$ of $V$ such that $w \in A \backslash B$ and $w \in \operatorname{Lin}((A \backslash\{w\}) \cup\{v\})$.

(13) Let $F$ be a field, $V$ be a vector space over $F$, and $A$ be a subset of $V$. Suppose $A$ is linearly independent. Let $a$ be an element of $V$. If $a \notin$ the carrier of $\operatorname{Lin}(A)$, then $A \cup\{a\}$ is linearly independent. 
Let $F$ be a field and let $V$ be a vector space over $F$. Observe that the matroid of linearly independent subsets of $V$ has exchange property.

Let $F$ be a field and let $V$ be a finite dimensional vector space over $F$. Note that the matroid of linearly independent subsets of $V$ is finite-membered.

\section{Maximal Independent Subsets, Ranks, and Basis}

Let $M$ be a subset family structure and let $A, C$ be subsets of $M$. We say that $A$ is maximal independent in $C$ if and only if:

(Def. 10) $A$ is independent and $A \subseteq C$ and for every subset $B$ of $M$ such that $B$ is independent and $B \subseteq C$ and $A \subseteq B$ holds $A=B$.

The following propositions are true:

(14) Let $M$ be a non void finite-degree subset family structure and $C, A$ be subsets of $M$. Suppose $A \subseteq C$ and $A$ is independent. Then there exists an independent subset $B$ of $M$ such that $A \subseteq B$ and $B$ is maximal independent in $C$.

(15) Let $M$ be a non void finite-degree subset-closed subset family structure and $C$ be a subset of $M$. Then there exists an independent subset of $M$ which is maximal independent in $C$.

(16) Let $M$ be a non empty non void subset-closed finite-degree subset family structure. Then $M$ is a matroid if and only if for every subset $C$ of $M$ and for all independent subsets $A, B$ of $M$ such that $A$ is maximal independent in $C$ and $B$ is maximal independent in $C$ holds card $A=\operatorname{card} B$.

Let $M$ be a finite-degree matroid and let $C$ be a subset of $M$. The functor Rnk $C$ yields a natural number and is defined by:

(Def. 11) $\operatorname{Rnk} C=\bigcup\{\operatorname{card} A ; A$ ranges over independent subsets of $M: A \subseteq C\}$.

One can prove the following propositions:

(17) Let $M$ be a finite-degree matroid, $C$ be a subset of $M$, and $A$ be an independent subset of $M$. If $A \subseteq C$, then card $A \leq \operatorname{Rnk} C$.

(18) Let $M$ be a finite-degree matroid and $C$ be a subset of $M$. Then there exists an independent subset $A$ of $M$ such that $A \subseteq C$ and card $A=\operatorname{Rnk} C$.

(19) Let $M$ be a finite-degree matroid, $C$ be a subset of $M$, and $A$ be an independent subset of $M$. Then $A$ is maximal independent in $C$ if and only if $A \subseteq C$ and card $A=\operatorname{Rnk} C$.

(20) For every finite-degree matroid $M$ and for every finite subset $C$ of $M$ holds $\operatorname{Rnk} C \leq \operatorname{card} C$.

(21) Let $M$ be a finite-degree matroid and $C$ be a finite subset of $M$. Then $C$ is independent if and only if $\operatorname{card} C=\operatorname{Rnk} C$.

Let $M$ be a finite-degree matroid. The functor $\operatorname{Rnk} M$ yielding a natural number is defined by: 
(Def. 12) $\operatorname{Rnk} M=\operatorname{Rnk}\left(\Omega_{M}\right)$.

Let $M$ be a non void finite-degree subset family structure. An independent subset of $M$ is said to be a basis of $M$ if:

(Def. 13) It is maximal independent in $\Omega_{M}$.

One can prove the following propositions:

(22) For every finite-degree matroid $M$ and for all bases $B_{1}, B_{2}$ of $M$ holds $\operatorname{card} B_{1}=\operatorname{card} B_{2}$.

(23) For every finite-degree matroid $M$ and for every independent subset $A$ of $M$ there exists a basis $B$ of $M$ such that $A \subseteq B$.

We follow the rules: $M$ is a finite-degree matroid, $A, B, C$ are subsets of $M$, and $e, f$ are elements of $M$.

Next we state four propositions:

(24) If $A \subseteq B$, then Rnk $A \leq \operatorname{Rnk} B$.

(25) $\operatorname{Rnk}(A \cup B)+\operatorname{Rnk}(A \cap B) \leq \operatorname{Rnk} A+\operatorname{Rnk} B$.

(26) Rnk $A \leq \operatorname{Rnk}(A \cup B)$ and $\operatorname{Rnk}(A \cup\{e\}) \leq \operatorname{Rnk} A+1$.

(27) If $\operatorname{Rnk}(A \cup\{e\})=\operatorname{Rnk}(A \cup\{f\})$ and $\operatorname{Rnk}(A \cup\{f\})=\operatorname{Rnk} A$, then $\operatorname{Rnk}(A \cup\{e, f\})=\operatorname{Rnk} A$.

\section{Dependence on a Set, Spans, and Cycles}

Let $M$ be a finite-degree matroid, let $e$ be an element of $M$, and let $A$ be a subset of $M$. We say that $e$ is dependent on $A$ if and only if:

(Def. 14) $\operatorname{Rnk}(A \cup\{e\})=\operatorname{Rnk} A$.

We now state two propositions:

(28) If $e \in A$, then $e$ is dependent on $A$.

(29) If $A \subseteq B$ and $e$ is dependent on $A$, then $e$ is dependent on $B$.

Let $M$ be a finite-degree matroid and let $A$ be a subset of $M$. The functor Span $A$ yielding a subset of $M$ is defined as follows:

(Def. 15) $\operatorname{Span} A=\{e \in M: e$ is dependent on $A\}$.

Next we state several propositions:

(30) $e \in \operatorname{Span} A$ iff $\operatorname{Rnk}(A \cup\{e\})=\operatorname{Rnk} A$.

(31) $A \subseteq \operatorname{Span} A$.

(32) If $A \subseteq B$, then $\operatorname{Span} A \subseteq \operatorname{Span} B$.

(33) $\operatorname{Rnk} \operatorname{Span} A=\operatorname{Rnk} A$.

(34) If $e$ is dependent on $\operatorname{Span} A$, then $e$ is dependent on $A$.

(35) $\operatorname{Span} \operatorname{Span} A=\operatorname{Span} A$.

(36) If $f \notin \operatorname{Span} A$ and $f \in \operatorname{Span}(A \cup\{e\})$, then $e \in \operatorname{Span}(A \cup\{f\})$. 
Let $M$ be a subset family structure and let $A$ be a subset of $M$. We say that $A$ is cycle if and only if:

(Def. 16) $A$ is dependent and for every element $e$ of $M$ such that $e \in A$ holds $A \backslash\{e\}$ is independent.

Next we state the proposition

(37) If $A$ is cycle, then $A$ is non empty and finite.

Let us consider $M$. Note that every subset of $M$ which is cycle is also non empty and finite.

One can prove the following propositions:

(38) $A$ is cycle iff $A$ is non empty and for every $e$ such that $e \in A$ holds $A \backslash\{e\}$ is maximal independent in $A$.

(39) If $A$ is cycle, then $\operatorname{Rnk} A+1=\overline{\bar{A}}$.

(40) If $A$ is cycle and $e \in A$, then $e$ is dependent on $A \backslash\{e\}$.

(41) If $A$ is cycle and $B$ is cycle and $A \subseteq B$, then $A=B$.

(42) If for every $B$ such that $B \subseteq A$ holds $B$ is not cycle, then $A$ is independent.

(43) If $A$ is cycle and $B$ is cycle and $A \neq B$ and $e \in A \cap B$, then there exists $C$ such that $C$ is cycle and $C \subseteq(A \cup B) \backslash\{e\}$.

(44) If $A$ is independent and $B$ is cycle and $C$ is cycle and $B \subseteq A \cup\{e\}$ and $C \subseteq A \cup\{e\}$, then $B=C$.

\section{REFERENCES}

[1] Broderick Arneson and Piotr Rudnicki. Recognizing chordal graphs: Lex BFS and MCS. Formalized Mathematics, 14(4):187-205, 2006.

[2] Grzegorz Bancerek. Cardinal numbers. Formalized Mathematics, 1(2):377-382, 1990

[3] Grzegorz Bancerek. The fundamental properties of natural numbers. Formalized Mathematics, 1(1):41-46, 1990.

[4] Grzegorz Bancerek. Tarski's classes and ranks. Formalized Mathematics, 1(3):563-567, 1990.

[5] Czesław Byliński. Functions and their basic properties. Formalized Mathematics, 1(1):5565, 1990.

[6] Czesław Byliński. Functions from a set to a set. Formalized Mathematics, 1(1):153-164, 1990.

[7] Czesław Byliński. Some basic properties of sets. Formalized Mathematics, 1(1):47-53, 1990.

[8] Agata Darmochwał. Finite sets. Formalized Mathematics, 1(1):165-167, 1990.

[9] Mariusz Giero. Hierarchies and classifications of sets. Formalized Mathematics, 9(4):865869, 2001.

[10] Zbigniew Karno. The lattice of domains of an extremally disconnected space. Formalized Mathematics, 3(2):143-149, 1992.

[11] Eugeniusz Kusak, Wojciech Leończuk, and Michał Muzalewski. Abelian groups, fields and vector spaces. Formalized Mathematics, 1(2):335-342, 1990.

[12] Witold Lipski. Kombinatoryka dla programistów, chapter Matroidy, pages 163-169. Wydawnictwo Naukowo-Techniczne, 1982.

[13] Robert Milewski. Associated matrix of linear map. Formalized Mathematics, 5(3):339345,1996

[14] Adam Naumowicz. On Segre's product of partial line spaces. Formalized Mathematics, $9(2): 383-390,2001$ 
[15] Beata Padlewska. Families of sets. Formalized Mathematics, 1(1):147-152, 1990.

[16] Beata Padlewska and Agata Darmochwał. Topological spaces and continuous functions. Formalized Mathematics, 1(1):223-230, 1990.

[17] Andrzej Trybulec. Domains and their Cartesian products. Formalized Mathematics, 1(1):115-122, 1990.

[18] Wojciech A. Trybulec. Basis of vector space. Formalized Mathematics, 1(5):883-885, 1990.

[19] Wojciech A. Trybulec. Partially ordered sets. Formalized Mathematics, 1(2):313-319, 1990.

[20] Wojciech A. Trybulec. Subspaces and cosets of subspaces in vector space. Formalized Mathematics, 1(5):865-870, 1990.

[21] Wojciech A. Trybulec. Vectors in real linear space. Formalized Mathematics, 1(2):291-296, 1990.

[22] Zinaida Trybulec. Properties of subsets. Formalized Mathematics, 1(1):67-71, 1990.

[23] D. J. A. Welsh. Matroid theory. Academic Press, London, New York, San Francisco, 1976.

Received July 30, 2008 